\title{
Design and Implementation Pulse Compression for S-Band Surveillance Radar in FPGA
}

\author{
Kalfika Yani, Fiky Y. Suratman and Koredianto Usman \\ ${ }^{1}$ School of Electrical Engineering, Telkom University, Bandung, 40287, Indonesia
}

Manuscript received April 19, 2020; revised October 10, 2020; accepted December 2, 2020

\begin{abstract}
The air surveillance radar system consists of four main parts, antenna, RF front-end, radar signal processing, and radar data processing. The S-band radar works in $2.7 \mathrm{GHz}$ to $3.1 \mathrm{GHz}$. The radar waveform consists of two types of waveform signals, continuous wave $(\mathrm{CW})$ and pulse compression. At the same power transmit level, pulse compression has longer transmission range than $\mathrm{CW}$, because the signal only transmits in short pulse. In the modern radar, waveform is implemented in digital platform. With digital platform, the radar waveform can be optimized without developing the new hardware platform. In this research we have designed and simulated a radar signal processing from baseband to IF using Xilinx ML-605 platform combined with FMC-150 high speed ADC/DAC. Field Programmable Gate Array (FPGA) is the best platform to implemented radar signal processing, because FPGA has ability to work in high speed data rate and parallel processing. The result shows that the implementation of pulse compression radar waveform on ML-605 and FMC150, taped on Chipscope debugger, is matched with Matlab simulation result. With 400 sample of chirp signals, the output matched filter has maximum value on $400^{\text {th }}$ sample, both on simulation and implementation. This research can implement to pulse compression S-band surveillance radar with 2.7 to $3.1 \mathrm{GHz}$.
\end{abstract}

Keywords: pulse compression; matched filter; FPGA; ML-605; FMC-150

doi: 10.25124/jmecs.v7i1.2631

\section{Introduction}

Existing radar system implements full hardware from baseband to radio frequency (RF). This technique is not efficient since one change in the system requirement should be followed by alteration of whole hardware. This process is also time consuming and pricey due to verification process of new design and new hardware development.

Implementing digital signal processing might provide a compact system. It requires few hardware rather than full hardware implementation which needs more space and components to produce radar waveform from baseband to intermediate frequency (IF). Implementation waveform radar using FPGA is more efficient than using hardware, because we can modify the waveform based on different specification without develop the new hardware. It makes efficient on time, dimension and budget. By using FPGA, the system only needs an integrated circuit and few additional components to create similar radar waveform.

Previous works show that the Virtex ${ }^{\circledR}-6$ FPGA ML605 Evaluation Kit [1] has been used developing system designs that demand high-performance, serial connectivity and advanced memory interfacing. In addition, integrated tools help streamline the creation of elegant solutions to complex design requirements [2].

In this study, we have designed and simulated a radar signal processing from baseband to IF using Xilinx ML-605 platform which combined with FMC150 high speed ADC/DAC. The FPGA simulation is found to be consistent with the implementation and the resulted waveform can be implemented on S-band.

\section{System Modelling}

\subsection{Radar Signal Processing System}

Radar signal processing consist of two parts, they are transmitter $(\mathrm{Tx})$ and receiver $(\mathrm{Rx})$, as in Figure 1. In Tx generator, there are information signal of pulse compression (chirp) in complex number. Chirp signal is in baseband frequency. Chirp signal processed in Digital Up Converter (DUC), to translate chirp signal from baseband frequency to Intermediate Frequency (IF).

In Tx generator, there are information signal of pulse compression (chirp) in complex number. Chirp signal is in baseband frequency. Chirp signal processed in Digital Up Converter (DUC), to translate chirp signal from baseband frequency to Intermediate Frequency (IF). 

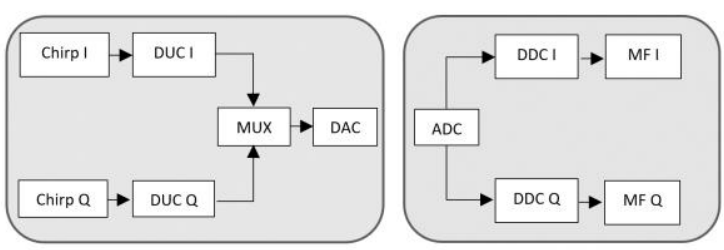

Figure 1: Radar Signal Processing Tx-Rx

In this research the NLFM equation is obtained by mirroring the Linear Frequency Modulation (LFM) signal. Both LFM and NLFM equations are presented in Eq. (1) and (2), respectively, where $\beta$ is the bandwidth in $\mathrm{Hz}, \mathrm{t}$ is the timing in second, and $\tau$ is the period.

$$
x(t)=e^{j \pi \beta t^{2} / \tau}=e^{j \theta(t)}, 0 \leq t \leq \tau
$$

The NLFM in this research is by mirroring the LFM signal, as in (2).

$$
x(t)=e^{j \pi\left(\left(\frac{\beta}{\tau}\right) *\left(t-\frac{\tau}{2}\right)\right)^{2}}=e^{j \theta(t)}, 0 \leq t \leq \tau
$$

The baseband signal work in $2 \mathrm{MHz}$ of sampling frequency, and the Intermediate Frequency (IF) sampling frequency is $240 \mathrm{MHz}$. To translate the sampling frequency from $4 \mathrm{MHz}$ to $240 \mathrm{MHz}$, it needs to implement Digital Up Converter (DUC). DUC consist of up-sampling to increase the sampling frequency from $4 \mathrm{MHz}$ to $240 \mathrm{MHz}$, and mixer the baseband signal with IF frequency, which is $60 \mathrm{MHz}$. The IF signal sends through the ADC with minimum sampling $240 \mathrm{MHz}$.

In the receiver, the IF signal will come from ADC with sampling frequency $240 \mathrm{MHz}$. In receiver there are two process, Digital Down Converter (DDC) and Matched Filter. The DDC process consist of two process, down conversion from $240 \mathrm{MHz}$ of the IF signal to $4 \mathrm{MHz}$ baseband signal. The output of DDC will process in Matched Filter (MF) to get the receive signal.

The Virtex®-6 FPGA ML605 Evaluation Kit [1] is the Xilinx base platform for developing system designs that demand high-performance, serial connectivity and advanced memory interfacing. This yields design applications for markets such as wired telecommunications, wireless infrastructure, broadcast and many others. Integrated tools help streamline the creation of elegant solutions to complex design requirements [2].

The FMC150 is a dual channel ADC and dual channel DAC FMC daughter card. The card provides two 14-bit A/D channels and two 16-bit D/A channels. [3].

\subsection{System Design}

The system is designed in Matlab/Simulink with the requirements as in Table 1. There are three main parts on transmitter, developed chirp Inphase (I) and chirp Quadrature (Q), upsampling process, carrier source (sine and cosine waves), mixer, and IQ process, as in Figure 2. On the receiver, there are three main process, mixer, downsampling process, and matched filtering as in Figure 3. The chirp signal is modelling in matlab. To implement in FPGA, the complex signal separate to real (Inphase) and imaginer (Quadrature) part, as in Figure 4.

Table 1. System requirements

\begin{tabular}{|l|l|}
\hline $\begin{array}{l}\text { Baseband Sampling } \\
\text { Frequency }\end{array}$ & $4 \mathrm{MHz}$ \\
\hline Bandwidth Signal & $2 \mathrm{MHz}$ \\
\hline $\begin{array}{l}\text { Pulse Repetition Interval } \\
(\mathrm{PRI})\end{array}$ & $1 \mathrm{~ms}$ \\
\hline Duty Cycle & $10 \%(100 \mu \mathrm{s})$ \\
\hline Chirp Phase & $\mathrm{NLFM}$ \\
\hline IF Sampling Frequency & $240 \mathrm{MHz}$ \\
\hline IF Carrier Frequency & $60 \mathrm{MHz}$ \\
\hline
\end{tabular}

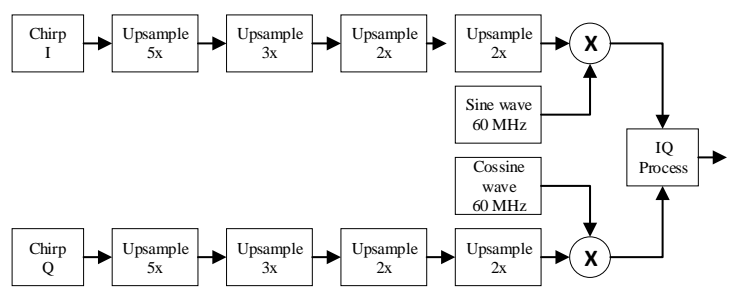

Figure 2: Transmitter

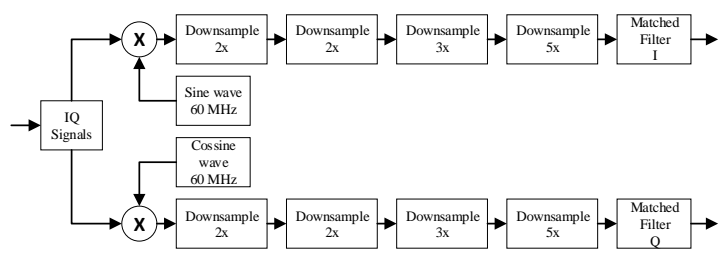

Figure 3: Receiver

The duty cycle of chirp signal is $10 \%$, with one PRI is $1 \mathrm{~ms}$ and the baseband sampling frequency is 4 $\mathrm{MHz}$. When transmit signal in one PRI, the sampling frequency is $1 \mathrm{~ms}=4000$ sample, and $10 \%$ from 4000 sample is 400 sample. The transmit chirp is 400 sample in one PRI, as in Figure 5. 


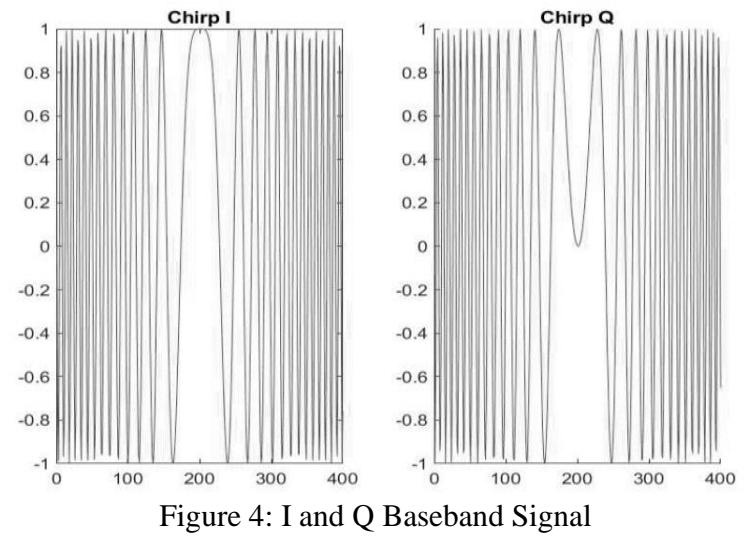

The baseband carrier frequency is $4 \mathrm{MHz}$, and the IF frequency is $240 \mathrm{MHz}$. To obtain $240 \mathrm{MHz}$ sampling frequency, the $4 \mathrm{MHz}$ baseband signal need to be multiplied up to 60 times. To void noisy results, this up-conversion process is conducted consecutively by multiplying with 5,3,2 and 2 times. The $4 \mathrm{MHz}$ output signal with $240 \mathrm{MHz}$ of sampling frequency, mix with $60 \mathrm{MHz}$ carrier signal with $240 \mathrm{MHz}$ of sampling frequency, as in Figure 6.

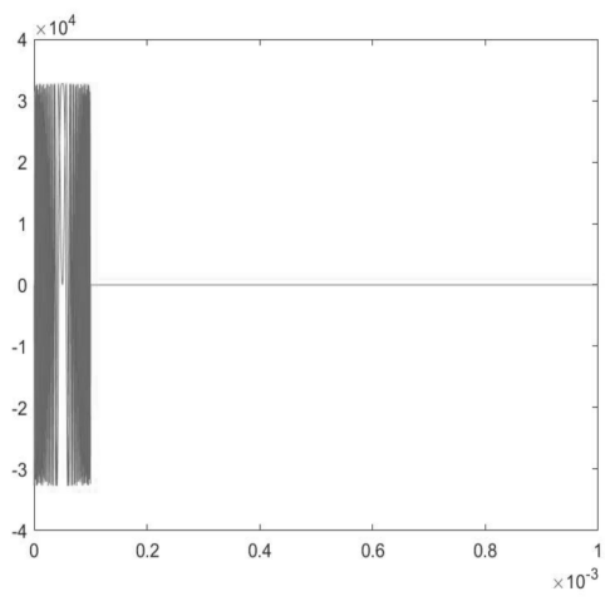

Figure 5: Chirp transmit in one PRI

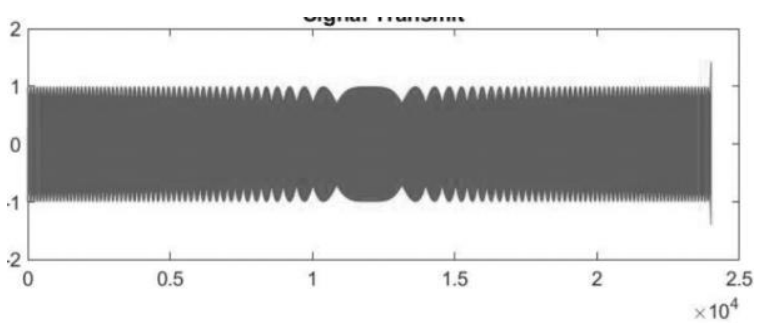

Figure 6: Transmit Signal In IF

Since the baseband signal is separate in I and Q, the carrier in IF is orthogonal signal consist of inphase (sinus) signals and quadrature (cosine) signals. In the receiver, the input ADC multiply with IF carrier, $60 \mathrm{MHz}$ sinus signals and $60 \mathrm{MHz}$ cosine signals with $240 \mathrm{MHz}$ sampling frequency. The $2 \mathrm{MHz}$ signal with $240 \mathrm{MHz}$ sampling frequency is divided by 5, 3, 2 and
2 times. The $2 \mathrm{MHz}$ baseband signal with $4 \mathrm{MHz}$ sampling frequency is processed in matched filter. The matched filter has filter coefficient which same with the chirp I and chirp Q in transmitter, as in Figure 7.

\section{System Implementation}

Xilinx ML-605 is development kit for Virtex-6 XC6VLX240T-1FFG1156 FPGA, which have specification as Table 2 [4].

To implement the radar signal processing, ML605 combined with the FMC-150 high speed ADC/DAC. The FMC150 is a four channel ADC/DAC FMC daughter card. The card provides two 14-bit A/D channels and two 16-bit D/A channels which can be clocked by an internal clock source (optionally locked to an external reference) or an externally supplied sample clock. In addition, there is one trigger input for customized sampling control. The FMC150 daughter card is mechanically and electrically compliant to FMC standard (ANSI/VITA 57.1). The FMC150 has a low-pin count connector, front panel $\mathrm{I} / \mathrm{O}$, and can be used in a conduction cooled environment. The ML-605 and FMC-150 show in Figure 8.

Table 1: Virtex-6 XC6VLX240T-1FFG1156 FPGA Specification

\begin{tabular}{|l|l|}
\hline Logic Cells & 241,152 \\
\hline Memory (Kb) & 14,976 \\
\hline DSP Slices & 768 \\
\hline Maximum Transceiver (GTX) & 24 \\
\hline Max User I/O & 720 \\
\hline
\end{tabular}

Floating point chirp from Matlab is convert to fixed point lookup table to implement in FPGA. There are two lookup table, chirp I and chirp Q. To validate if the implement signal is according to Matlab simulation, the implementation signal will debug with Chipscope as in Figure 9.

DUC and DDC filter from Matlab produce fixed point coefficient in .coe files. Filter is implement using this coefficient look up table. Sinus and cosinus waves carrier signal lookup table also generated from matlab. To validate if the $60 \mathrm{MHz}$ carrier is correct, the output of FMC150 DAC connected to Spectrum Analyzer, as in Figure 9.

The output DAC connect to the ADC input. The input signal mixed with Inphase (I) and Quadrature (Q) IF carrier signal. Output mixer will process in DDC to translate the sampling frequency from 240 $\mathrm{MHz}$ to $4 \mathrm{MHz}$. The final process in receiver of radar signal processing is matched filter. The matched filter coefficient lookup table generated by Matlab, and the number of coefficients is same with the magnitude of chirp signal in I and Q. The output matched filter I can show in Figure 11, and the output of matched filter Q is show in Figure 12. 


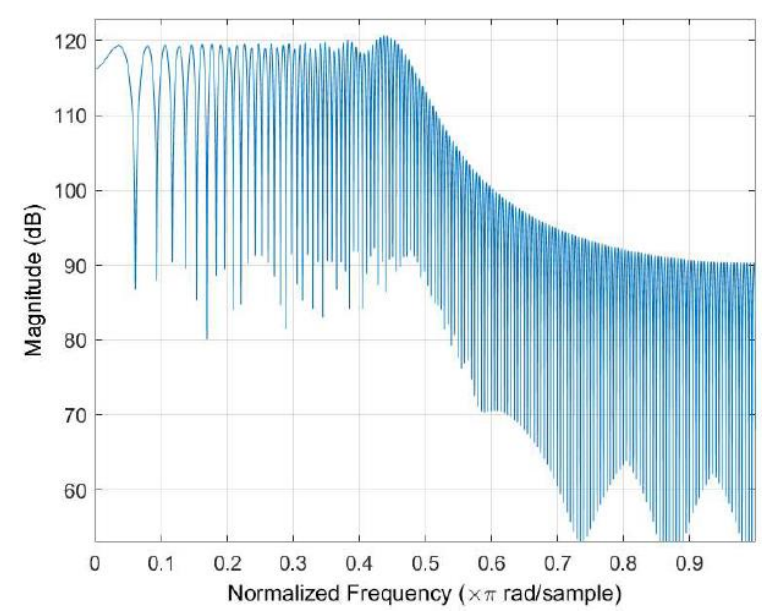

7(a)

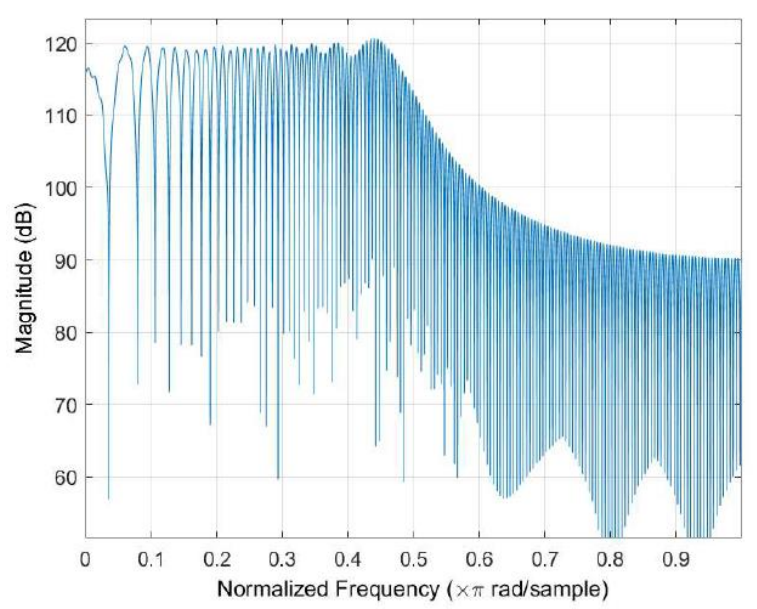

7(b)

Figure 7(a) Matched Filter I Response Filter and (b) Matched Filter Q Response Filter

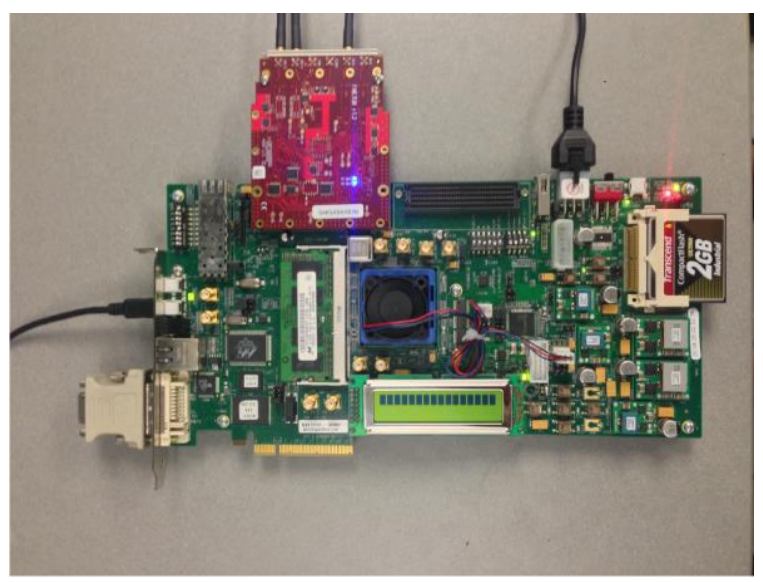

Figure 8: ML-605 and FMC-150

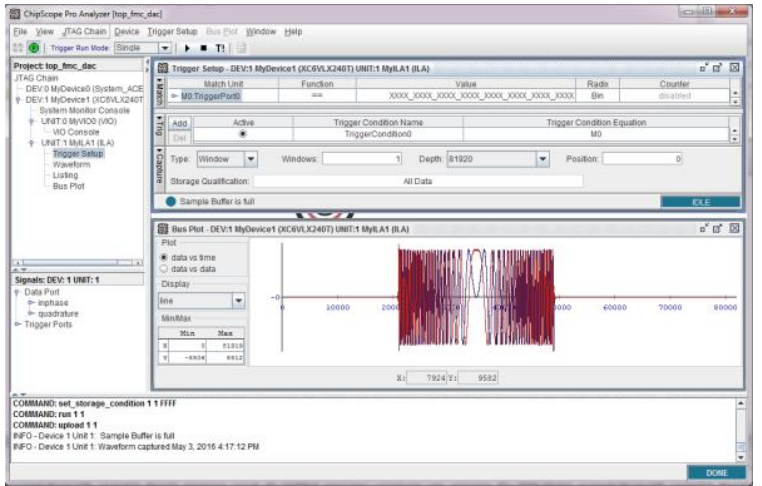

Figure 9: Chirp I and Q implementation in FPGA

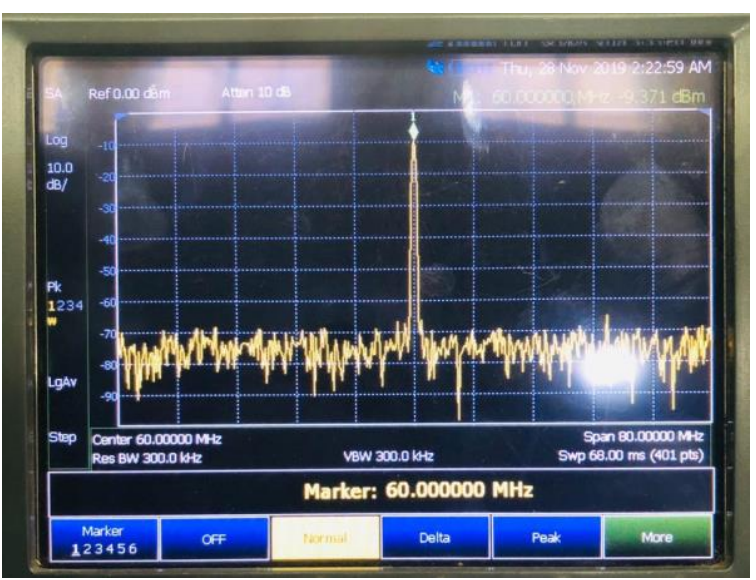

Figure 10: IF carrier signal on Spectrum Analyzer

\section{Conclusions}

The implementation of pulse compression radar waveform on ML-605 and FMC150, which taped on Chipscope debugger is matched with Matlab simulation. With 400 sample of chirp signals, the output matched filter has maximum value on $400^{\text {th }}$ sample, both on simulation and implementation. This research can be implemented for pulse compression S-band surveillance radar at 2.7 to $3.1 \mathrm{GHz}$. In the future work, this research can implement in FPGA with add noise, so the SNR can be calculated. The chirp can be changed to smaller duty cycle and calculate the timing process in FPGA. 


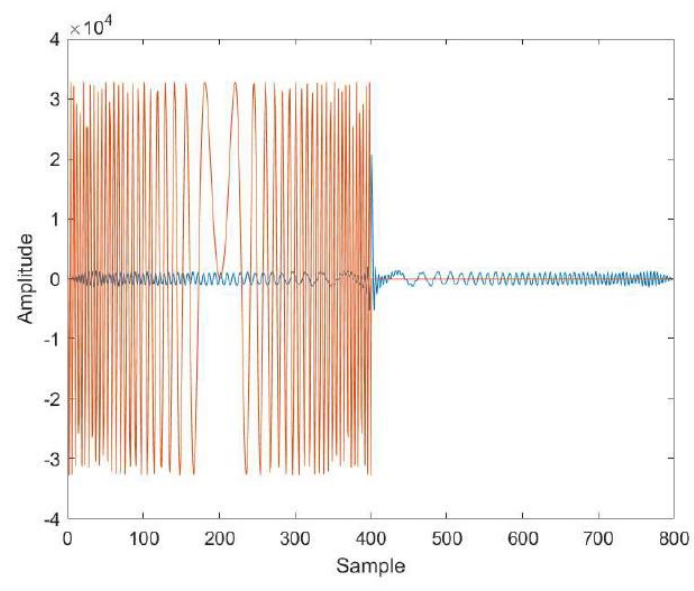

11(a)

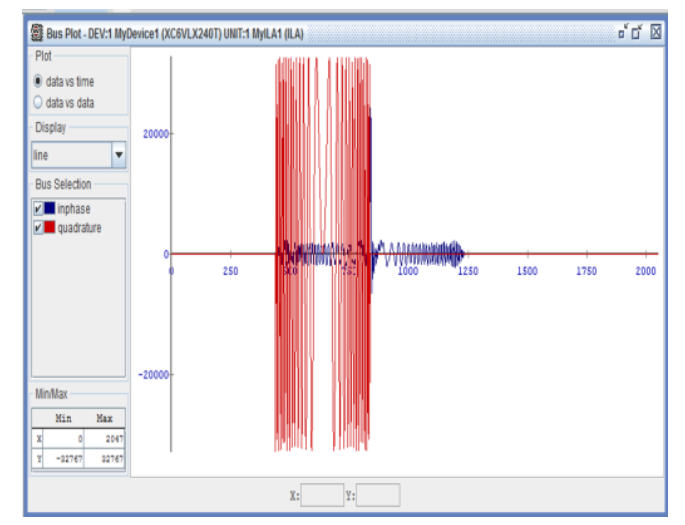

11(b)

Figure 11(a) Chirp (orange line) and Output (blue line) Matched Filter I Matlab Simulation and (b) Chirp and Output Matched Filter I FPGA Implementation on Chipscope.

\section{References}

[1] M. R. Widyantara, Sugihartono, F. Y. Suratman and P. D. Slamet Widodo, "Analysis of Non Linear Frequency Modulation (NLFM) Waveforms for Pulse Compression Radar," Jurnal Elektronika dan Telekomunikasi (JET), vol. 18, no. August 2018, pp. 27-34, 2018.

[2] Xilinx, "ML605 Reference Design User Guide," Xilinx, San Jose, California, US, September 25, 2009.

[3] Abaco, "https://www.abaco.com," Abaco, 2015. [Online]. Available: https://www.abaco.com/products/fmc150-fpgamezzanine-card.

[4] Xilinx, "www.xilinx.com," Xilinx, 2009. [Online]. Available: https://www.xilinx.com/products/boards-andkits/ek-v6-ml605-g.html\#hardware.

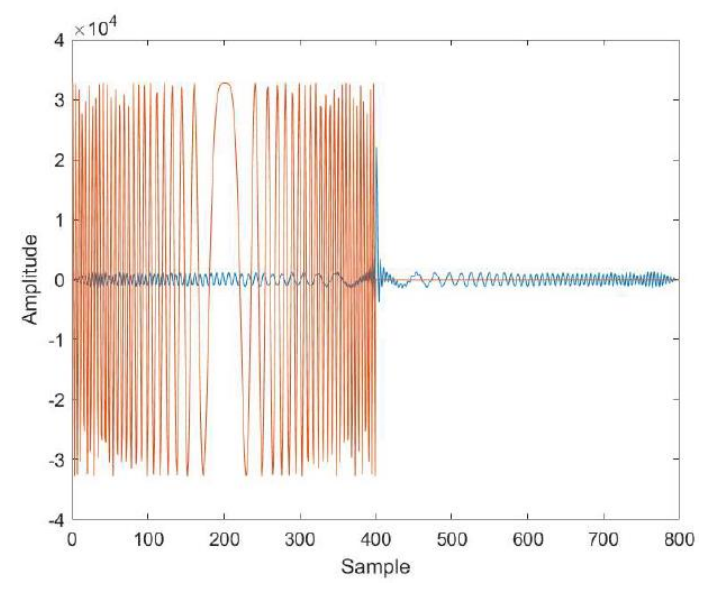

12(a)

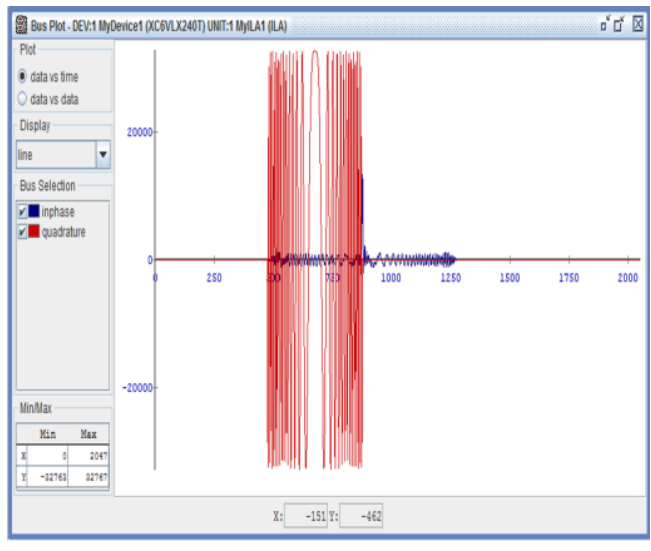

12(b)

Figure 12(a) Chirp (orange lines) and Output (blue lines) Matched Filter Q Matlab Simulation and (b) Chirp and Output Matched Filter Q FPGA Implementation on Chipscope

[5] N. u. Azim and W. Jun, "Hardware Optimized Implementation of Digital Pulse Compression Based on FPGA," 2016 International Conference on Radar, Antenna, Microwave, Electronics, and Telecommunications, 2016.

[6] J. J. Alter and J. O. Coleman, "Radar DIgital Signal Processing," in Radar Handbook, New York, McGrawHill, 2008, p. 25.

[7] M. Richards, Fundamentals of Radar Signal Processing, New York: Tata McGrawHill Education, 2005.

[8] Z. Yuxi, K. Li, W. Jun, S. Jinping and W. Zulin, "Methods and Experience Using Matlab and FPGA for Teaching Practice in Digital SIgnal Processing," International Conference on Education and Management Technology (ICEMET 2010), 2010. 
[9] M. I. Skolnik, Radar Handbook Third Edition, McGraw-Hill, 2008.

[10] B. R. Mahafza, Radar System Analysis and Design Using Matlab, McGraw-Hill, 2008.

[11] M. A. Richards, J. A. Scheer and W. A. Holm, Principle of Modern Radar: Basic Principles, volume 1, Institution of Engineering and Technology, 2010.

[12] M. T. Thin and M. S. S. Yi, "Pulse Compression Method for Radar Signal Processing," International Journal of Science and Engineering Applications, vol 3, 2014.

[13] C. Wolff, "radartutorial.eu," [Online]. Available: https://www.radartutorial.eu/08.transmitters/WaveformGenerator.en.html.

[14] M. Labitt, "Obtaining Low Sidelobes Using Non-Linear FM Pulse Compession," Lincoln Labolatory, Massachusetts Institute of Technology, Lexington, Massachusetts, 1994.

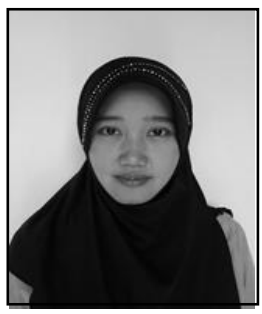

Kalfika Yani S.T., IPM., ASEAN Eng., was born on 15 September 1985. She graduated Bachelor Degree in 2008 from STT Telkom Bandung, Indonesia majoring in Telecommunication Engineering. During 2008-2020 she works in PT. Len Industri (Persero) as a Digital FPGA Engineer, and developed signal processing for Manpack Radio and Radar Signal Processing for 2D SBand Surveillance Radar and 3D Air Surveillance Radar.

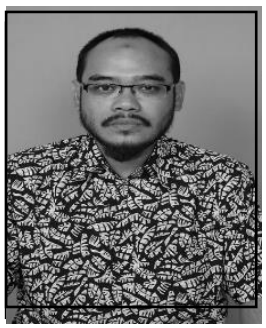

Dr. Ing Fiky Y. Suratman, S.T., M.T. was born in Jakarta, Indonesia, in 1976. He received Bachelor Degree from Engineering Physics and Master Degree from School of Electrical and Informatics (STEI), Institut Teknologi Bandung, Bandung, Indonesia in 1998 and 2006, respectively. He was awarded scholarship from German Academic Exchange Service (DAAD) to continue his study at Technische Universitaet Darmstadt (TU-Darmstadt), Germany, until he recived his Dr.-Ing. Degree (PhD) in 2014.
Early placement in industry (Astra Microtronics Technology) from 1998 to 2001, was then followed by lectureship in Universitas Komputer Indonesia (UNIKOM). In 2007, he joined the Faculty of Electrial Engineering, Institut Teknologi Telkom (now Universitas Telkom) as a faculty member. He is the head of Master Degree of Electrical Engineering, Telkom University since 2014. He is a member of IEEE (Signal Processing Soiciety and Communications Society), and since 2016 he is the chair of Signal Processing Society (SPS) Indonesia Chapter. His research interest lies on Statistical Signal Processing and Radar Signal Processing.

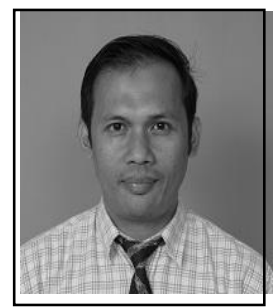

Dr. Koredianto Usman, S.T., M.Sc. was born in Sumatera Selatan Indonesia October 2, 1975. He graduated Bachelor Degree from Electrical Engineering Bandung Institute of Technology, Indonesia, 1999, and Master Degree from Munich Institute of Technology, Germany in 2001. In 2019, he obtained a PhD in Electrical and Informatics, Engineering from Bandung Technology Institute. His research interest is signal processing, and he has been publishing journal articles in various international proceedings and national journals. Besides, he is an active reviewer at international telecommunication conferences. 